\title{
Novel coronavirus (COVID-19) diagnosis using computer vision and artificial intelligence techniques: a review
}

\author{
Anuja Bhargava $^{1}$ (D) Atul Bansal ${ }^{1}$
}

Received: 21 June 2020 /Revised: 23 October 2020 / Accepted: 10 February 2021 /

Published online: 3 March 2021

(C) The Author(s), under exclusive licence to Springer Science+Business Media, LLC part of Springer Nature 2021

\begin{abstract}
The universal transmission of pandemic COVID-19 (Coronavirus) causes an immediate need to commit in the fight across the whole human population. The emergencies for human health care are limited for this abrupt outbreak and abandoned environment. In this situation, inventive automation like computer vision (machine learning, deep learning, artificial intelligence), medical imaging (computed tomography, X-Ray) has developed an encouraging solution against COVID-19. In recent months, different techniques using image processing are done by various researchers. In this paper, a major review on image acquisition, segmentation, diagnosis, avoidance, and management are presented. An analytical comparison of the various proposed algorithm by researchers for coronavirus has been carried out. Also, challenges and motivation for research in the future to deal with coronavirus are indicated. The clinical impact and use of computer vision and deep learning were discussed and we hope that dermatologists may have better understanding of these areas from the study.
\end{abstract}

Keywords Computer vision · Computed tomography · Machine learning · Coronavirus · COVID-19

\section{Introduction}

The Novel Coronavirus (Covid-19) named coronavirus because of the electronic microscope appearance to cosmic corona that is identical to the crown [15]. It is a widespread toxic disease epidemic in late 2019 and is originated from the serious syndrome of respiratory (SARS-COV2) [73] as shown in Fig. 1. It is a member of the "Coronaviradae family" that is non-

Anuja Bhargava anuja1012@gmail.com

Atul Bansal

atul.bansal@gla.ac.in

1 GLA University, Mathura, India 
Fig. 1 Example of SARS-COV-2

[73]

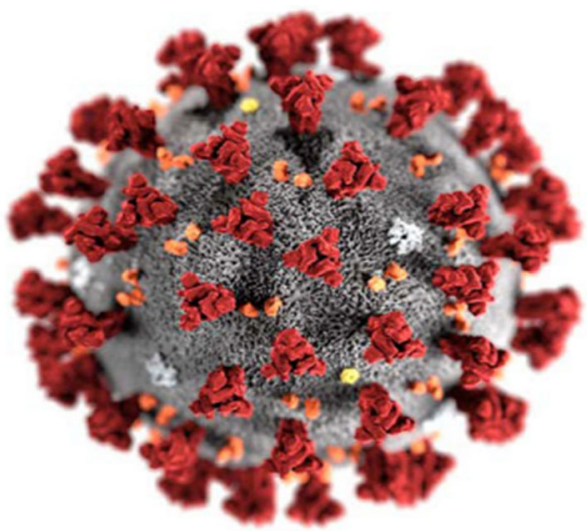

segmented, enveloped, and the sense viruses. SARS-COV-2 constitutes 14 bonded residues which precisely connect with "human angiotensin-converting enzyme 2 ". The breezy respiratory infection in humans is identified as COVID-19 as far as SARS-COV-2 identification is done. A various field like industry, supply chain, production, insurance, transport, agriculture, and tourism affected by COVID-19 results in shock of the economy globally [60] and is at high risk around the world. According to OECD (Organization for Economic Cooperation and Development) due to COVID-19, the economy this year is the lowest [71].

The first infection is noted in Wuhan [China] on December 31, 2019, having symptoms like fever, dry cough, fatigue, nausea, shortness of breath, lung infiltrates, and dyspnoea. All the cases were associated with the seafood market, like fish and animal-like bats, snakes, poultry, and marmots [67]. The WHO (World Health Organization) claims the PHEIC (Public Health Emergency of International Concern) on January 30, 2020, and recognized it as pandemic on March 11, 2020 [109-111]. The virus infects 38, 86,230 cases of COVID-19 [up to May 8, 2019] out of which $2,68,908$ reports death and 13,31,014 reports recovered. The geographical spread and various stats about recovery and deaths case of COVID-19 are shown in Figs. 2 and 3 till Oct 18, 2020.

The rise in several infections to people, results in curfew and lock-downs by governments all over the world and restricts people's movement, goods services and called for "work from home" to lower the virus spread rate. The governments are taking major steps to spread of virus globally. There is no vaccine claimed till now by any country for COVID- 19 .

Therefore, the researcher motivates to understand, explore, and treatments of COVID-19. The technologies such as computer vision and artificial intelligence could be best fitted in a crisis of COVID-19 [39, 69, 80]. The objective of this review paper is to give a comparative analysis of computer vision in COVID-19 and also to review various image acquisition, segmentation, and deep learning. This review is specified to US, Italy, Spain, Germany, Iran, France and India.

\section{Classification of key area}

This section presents different computer vision techniques used for COVID-19 briefly explained in subsequent sections, as depicted in Fig. 4. Also, the classification of positive and negative COVID-19 patients involves basic steps as shown in Fig. 5. 


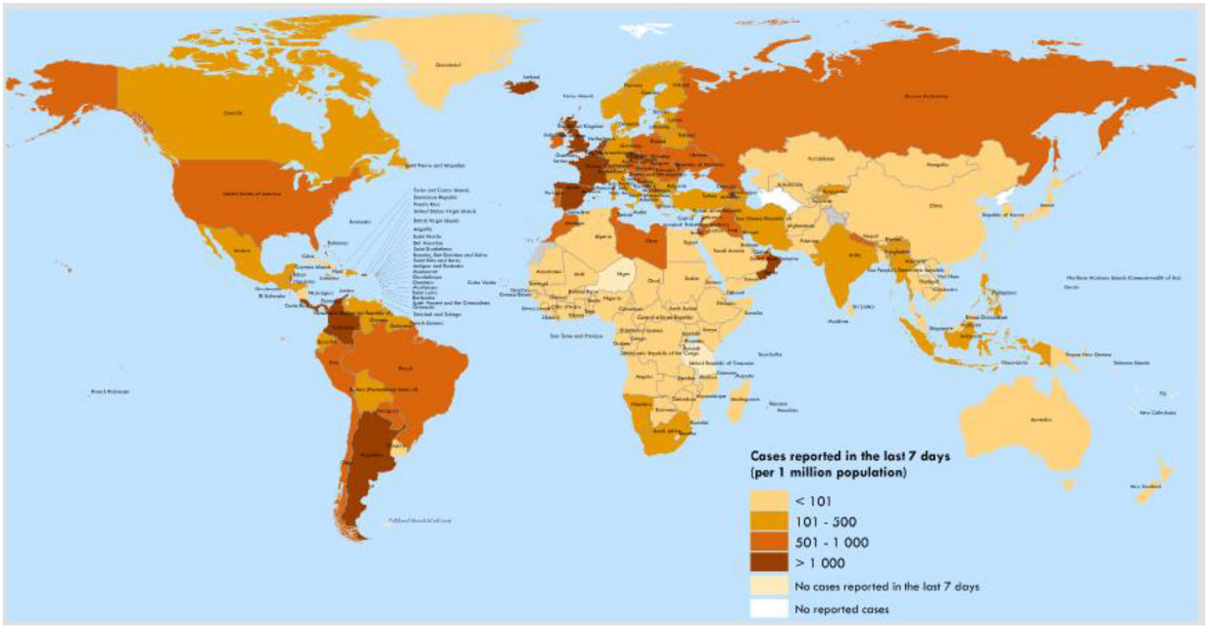

Fig. 2 Example of the geographical spread of confirmed COVID-19 till Oct 18 [109]

\subsection{Image acquisition}

"The judgment about the exact disease, especially after doctor examination is the diagnosis and expected development of the disease by a doctor is prognosis defined by Cambridge dictionary (https://dictionary.cambridge.org/). The COVID-19 currently diagnosis with RTqPCR (Reverse Transcriptase Quantitative Polymerase Chain Reaction) [103]. Nonetheless, this manual test is time-consuming and shows some of the false reports [20].

\subsubsection{Computed tomography (CT) scan}

Alternatively, acquiring an image using a CT scan of the chest obtains a more detailed, enhanced form compared to a standard x-ray scan. The images formed incorporate fats, bones,

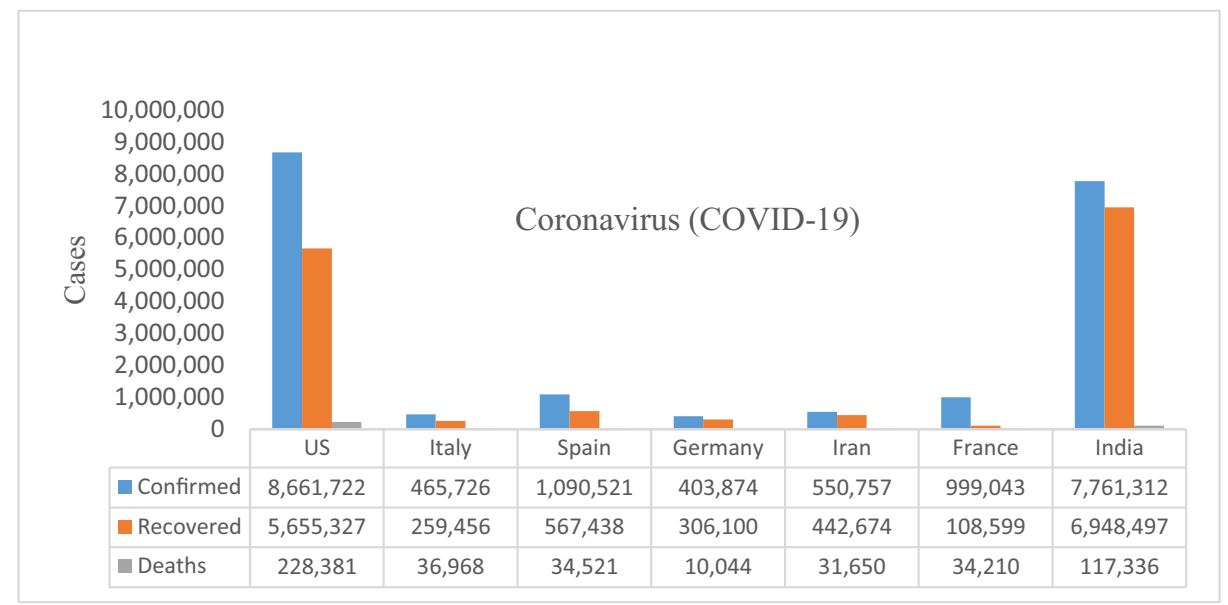

Fig. 3 Stats of COVID 19 in some countries till Oct, 18 [109] 


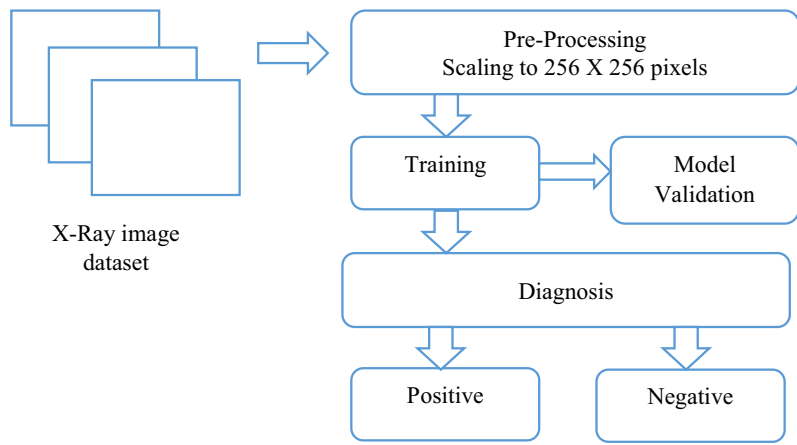

Fig. 4 Basic Steps to identify COVID-19 using image processing

organs, and muscles that result in accurate diagnose by physicians. The spiral chest CT and high-resolution scan are the two categories of CT scan [3]. Inspiral chest CT scan (https:// www.radiologyinfo.org/en/info.cfm?pg=chestct), the spiral lane is followed by an X-Ray tube, produces a 3D image of lungs. In a high-resolution scan, it produces an image from a single rotation by the X-Ray tube.

Crucial aspects of CT scan covers consolidation, ground-glass opacity, lesion distribution, nodules, and recitation of interlobular septa $[16,61,65]$. The radiologist's experts identify the disease which is time-consuming, therefore, to imbrute the process computer vision is used. Various images acquired by distinct researchers using CT scans comprise of subsequent characteristics as shown in Table 1. Figure 6 shows some of the samples from the CT scan images.

\subsubsection{X-ray image}

The digital CXR (Chest X-Ray) radiography is preferred for imaging because it is easily available and cheaper. It is us for cancer [77], cardiac [4] and osteoporosis disease [94]. Due to low contrast, pre-processing is done by contrast enhancement [47] in X-Ray imagery. Various images acquired by distinct researchers using X-Ray comprise of subsequent characteristics as sown in Table 2. Figure 7 shows some of the samples from the X-Ray scan images.

Various images acquired by distinct researchers using different resources comprise of subsequent characteristic as shown in Table 3.

Fig. 5 Basic platform of coronavirus

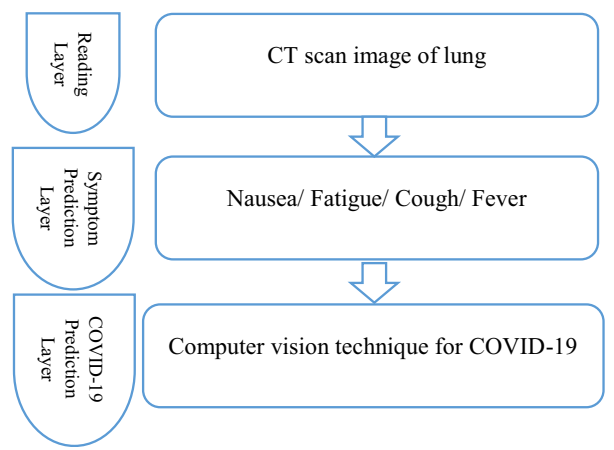


Table 1 Classical approaches for COVID-19 using CT-scan

\begin{tabular}{lllll}
\hline Authors & Database (CT Images) & Segmentation & No. of patient & Accuracy (\%) \\
\hline Jun Chen et al. [17] & 46,096 & UNet++ & 106 with 51+ve & $95.24 \%$ \\
Shuai Wang et al. [104] & 453 & - & 99 & $82.90 \%$ \\
Xiaowei Xu et al. [115] & 618 & VNet & 219 with 110+ve & $86.70 \%$ \\
Ying Song et al. [93] & 777 & & 88 & $86.00 \%$ \\
Opir Gozes et al. [34] & & U-Net & 56 & $99.60 \%$ \\
Fei Shan [85] & 249 & VB-Net & 249 & $91.60 \%$ \\
Cheng Jin et al. [43] & 970 & 2D CNN & 496 & $94.98 \%$ \\
Mucahid Barstuga et al. [9] & 150 & - & - & $99.68 \%$ \\
Lin Li [62] & 4356 & U-Net & 3322 & - \\
Chuansh Zheng [90] & - & U-Net & 540 & $95.90 \%$ \\
Shua Jin [44] & - & UNet++ & 1136 with $723+$ ve & - \\
\hline
\end{tabular}

\subsection{Image segmentation}

After acquiring an image, the segmentation of the image is a crucial fundamental step in the analysis and processing of the estimation of COVID-19. It depicts the ROI (Region of Interest) like infected lesions, lobes, lung in the image. The segmented ROI is used to extract features for analysis and diagnosis purposes. The immense quality 3-dimensional image is provided by a CT scan for disclosing COVID-19. The most prominent method for ROI in CT scan incorporates U-Net, U-Net++, VB-Net. Presently, none of the methods is refined for X-Ray image segmentation. Despite bounded segmentation methods, several researchers consider segmentation as a fundamental process to investigate COVID-19. Table 4 illustrates the segmentation technique involved in COVID-19.

The convolutional neural network $(\mathrm{CNN})$ to used to extract features from COVID-19 XRay images. In this process, a special type of CNN called a pre-trained model where the network is previously trained on the dataset, which contains millions of variety of images. So, transfer learning is applied by transferring weights that were already learned and reserved into the structure of the pre-trained model, such as Inception. The different types of features such as edges, texture, colors, and high-lighted patterns are extracted from the images.

\subsection{Diagnosis for COVID-19}

Artificial intelligence (AI) A dynamic tool for COVID-19 prediction and analysis is an Artificial Intelligence technique [108]. Presently, various researchers and their studies show AI has been widely utilized for COVID-19 issues using ML (Machine Learning) and DL
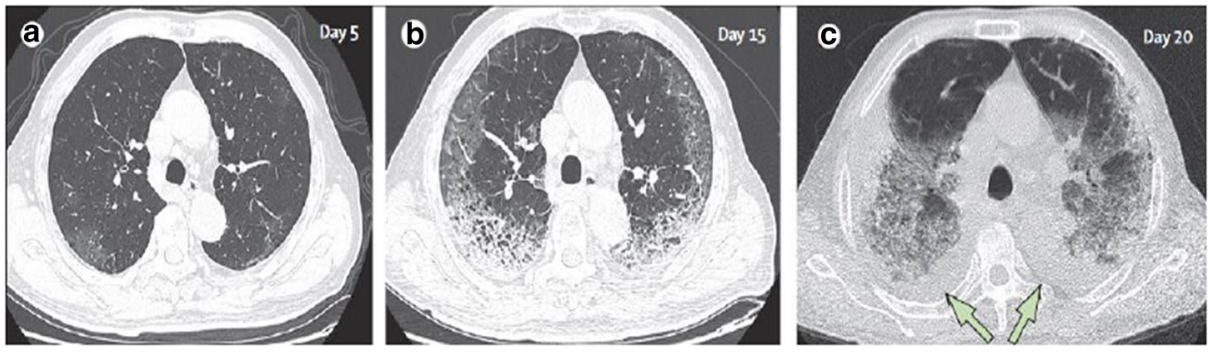

Fig. 6 After symptom of COVID19 a Day 5 b Day 15 c Day 20 
Table 2 Classical approaches for COVID-19 using X-Ray

\begin{tabular}{llll}
\hline Authors & Database (X-Ray Images) & Segmentation & Accuracy (\%) \\
\hline Guszt et al. [31] & 662 & U-Net+ & $97.50 \%$ \\
Asmaa et al. [1] & 80 & CNN & $95.12 \%$ \\
Ali Narin et al. [70] & - & ResNet50 & $97.00 \%$ \\
Linda Wang et al. [100] & 16,756 & - & $92.40 \%$ \\
Ezz El et al. [38] & - & DCNN & $89.00 \%$ \\
Khalid et al. [8] & 5856 & ResNet50 & $96.00 \%$ \\
Prabira et al. [84] & - & ResNet50 & $95.38 \%$ \\
Ioannis D et al. [6] & 1427 & VGG19 & $95.57 \%$ \\
Biraja Ghoshal et al. [33] & 68 & BCNN & $88.39 \%$ \\
Mohd F., Abul Hafeez [28] & - & ResNet50 & $96.23 \%$ \\
\hline
\end{tabular}

(Deep Learning). Generally, ML is used for précising the structure of the data which is handled by people [10]. ML methods train the input data and analyze the output data statistically. The application of ML includes the detection of infected persons and the temperature of the person $[5,12,27]$.

DL has been designed for better performance of neural networks and a type of artificial neural network and has multilayers. The more layers are increased, the greater accuracy is achieved. In machine learning, Deep Belief Networks (DBN) is a productive graphical model or, alternatively, a class of deep neural networks consisting of multiple layers in hidden nodes. When trained on a series of unsupervised examples, the DBN can learn to reconfigure its entries as probabilistic. The layers then act as feature detectors. After this learning phase, a DBN can be trained with more control to make the classification. DBNs can be seen as a 4 combination of simple, unsupervised networks, such as restricted Boltzmann machines (RBMs) or auto encoder, which serve as the hidden layer of each subnet, the visible layer of the next layer.

DL algorithms are used to detect solutions for COVID-19. The manifold neural network layers are used by connected weight vector [78, 92]. Various application of DL includes computer vision, object detection, speech recognition [42, 64, 68]. Several DL based solution for COVID-19 are established by AI methods [30]. Table 5 utilizes the literature related to the AI diagnosis of COVID-19.

The different companies utilized AI for adequate detection and disease diagnosis caused by a coronavirus. Table 6 shows some of the cases used by AI. The literature presents various AI-based solutions for COVID-19 detection using temperature detection of face $[82,98]$ and data analysis $[83$, 88].

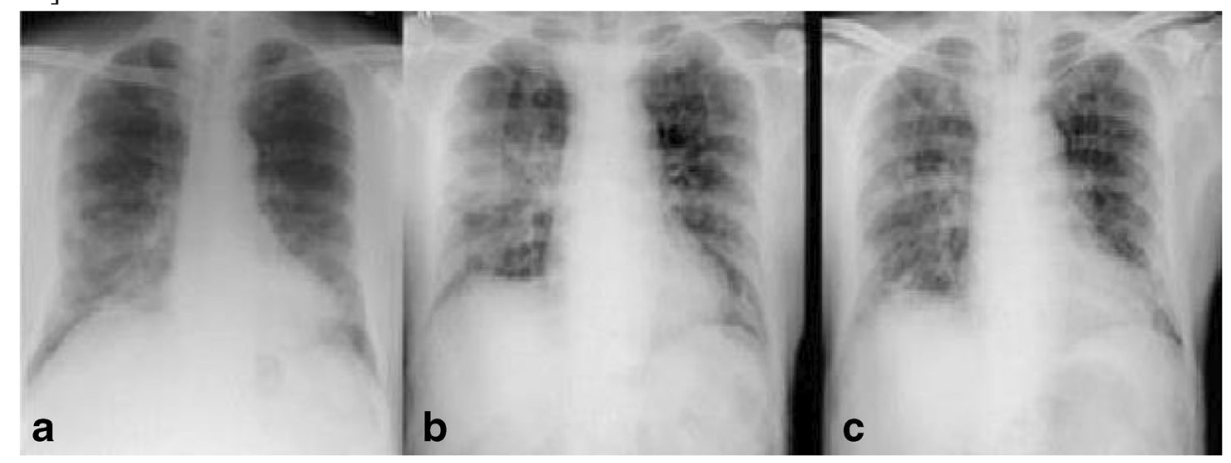

Fig. 7 Chest radiography a Day 0 b Day 4 c Day 7 
Table 3 Summary of the dataset available

\begin{tabular}{|c|c|c|}
\hline Dataset & $\begin{array}{l}\text { No. of } \\
\text { Images }\end{array}$ & Link \\
\hline $\begin{array}{l}\text { COVID CT DATASET } \\
\text { [123] }\end{array}$ & 349 & https://github.com/UCSD-AI4H/COVID-CT \\
\hline RADIOGRAPHY [22] & 2905 & https://github.com/tawsifur/COVID-19-Chest-X-ray-Detection \\
\hline IMAGE DATA [23] & & https://github.com/ieee8023/covid-chestxray-dataset. \\
\hline COVIDx Dataset [100] & 16,756 & https://github.com/ieee8023/covid-chestxray-dataset: \\
\hline ChestX-ray8 [101] & & https://nihcc.app.box.com/v/ChestXray-NIHCC). \\
\hline $\begin{array}{l}\text { Masked Face } \\
\quad \text { Recognition [105] }\end{array}$ & 90,000 & $\begin{array}{l}\text { https:/github. } \\
\text { com/X-zhangyang/Real-World-Masked-Face-Dataset. }\end{array}$ \\
\hline Thermal Image [58] & & - \\
\hline
\end{tabular}

\subsection{Avoidance and management}

Various guidelines [112] are granted by WHO for the prevention and control of COVID-19. Extensive strategies to restrain incorporates source control, early recognition, precautions, engineering, and control. To reduce the infection in the early stages, the utilization of vigilant equipment or mask is necessary. All over the world, some of the countries like India implement it as a jurisdiction strategy and was promoted by machine learning systems. The table utilizes the work related to the prevention and control of the disease (Table 7).

\subsection{Learning lessons COVID-19}

The coronavirus has been efficient and transparent, still, various points must be learned for the outbreak in the future utilized in Table 8.

\subsection{Infection syndrome and medication}

To date, there is no specific cure for infection originated by COVID-19. Yet, bountiful manifestation can be evaluated depending upon the condition of the patient. Currently, various researchers and teams are working on devise vaccination for the infection. Computer vision is

Table 4 Utilization of image segmentation techniques for COVID-19

\begin{tabular}{llll}
\hline Authors & Segmentation Tech. & ROI & Utilization \\
\hline Zheng et al. [124] & U Net & Lung & Examination \\
Cao et al. [13] & U Net & Lesion/ Lung & Evaluation \\
Huang et al. [40] & U Net & Lung lobes/ Lesion/ Lung & Evaluation \\
Qi et al. [79] & U Net & Lung lobes/ Lesion & Evaluation \\
Gozes et al. [35] & U Net & Lesion/ Lung & Examination \\
Li et al. [63] & U Net & Lesion & Examination \\
Chen et al. [18] & UNet++ & Lesion & Examination \\
Jin et al. [45] & UNet++ & Lesion/Lung & Examination \\
Shan et al. [86] & VB-Net & Lung lobes/ Lesion/ Lung & Evaluation \\
Tang et al. [96] & Commercial Software & Lesion/Lung & Evaluation \\
Shen et al. [89] & Threshold-based region growing & Lesion & Evaluation \\
\hline
\end{tabular}


Table 5 Study related to AI diagnosis

\begin{tabular}{|c|c|c|c|}
\hline Author & Dataset & Technique & Accuracy \\
\hline Ghoshal et al. [33] & 70 & $\mathrm{CNN}$ & $92.90 \%$ \\
\hline Narin et al. [70] & $50+$ ve, 50 -ve & ResNet50 & $98.00 \%$ \\
\hline Zhang et al. [122] & $70+$ ve, 1008 other & ResNet & $95.20 \%$ \\
\hline Wang et al. [100] & $45+$ ve, 1203 -ve & $\mathrm{CNN}$ & $83.50 \%$ \\
\hline Chen et al. [18] & $51+\mathrm{ve}, 51-\mathrm{ve}$ & UNet++ & $95.20 \%$ \\
\hline Zheng et al. [124] & $313+$ ve, 229 other & UNet, CNN & $95.90 \%$ \\
\hline Jin et al. [46] & $496+$ ve, 1385 other & $\mathrm{CNN}$ & - \\
\hline Jin et al. [45] & $723+$ ve, 413 other & UNet++, CNN & - \\
\hline Wang et al. [106] & $44+$ ve, 55 -ve & $\mathrm{CNN}$ & $82.90 \%$ \\
\hline Ying et al. [117] & $88+v e, 86$-ve & ResNet50 & $86.00 \%$ \\
\hline Xu et al. [116] & $219+\mathrm{ve}, 175$-ve & $\mathrm{CNN}$ & $86.70 \%$ \\
\hline Li et al. [63] & $468+v e, 1445$-ve & ResNet 50 & - \\
\hline Shi et al. [91] & $1658+v e, 1027$-ve & $\mathrm{RF}$ & $87.90 \%$ \\
\hline Tang et al. [97] & $176+v e$ & $\mathrm{RF}$ & $87.50 \%$ \\
\hline
\end{tabular}

the source of how helping to develop and support clinical management. The identification of crucial patients and their medical treatment is a major step in clinical management. A corona score (disease progression score) is suggested to adjust and classify the patient as shown in Fig. 8. "Corona score measures the progression of the patient over time. The corona score is computed by a volumetric summation of the network activation maps" [34]. Table 9 utilizes the work related to infected disease management and control.

\subsection{Provocation and future work}

In this paper, the challenges in research are highlighted first and then future directions are explored one by one.

\subsubsection{Provocation}

(i) Managerial Consideration- The use of computer vision (machine learning, deep learning) in the clinical sector like COVID-19 must be examined delicately.

Table 6 Cases used by AI

\begin{tabular}{lll}
\hline Use case & Developed/Used by & Aim \\
\hline Bluedot & Toronto-based Start-up & - Detects epidemics [11] \\
& & - Build a prediction model for virus detection \\
& & - Collect information by NLP and ML from social media \\
& & Government documents \\
Infravision & Tongji Hospital Wuhan & Healthcare data \\
& & - Detect disease precisely [41] \\
Alphafold & & - Early detection of patient \\
& & - SARS-COV-2 prediction [24] \\
& & - Entrusted by DL and ML \\
NVIDIA & Zhongnan Hospital, Wuhan & - Not verified yet \\
& & - Primarily used for detecting cancer \\
& & - identifies signs of COVID-19 [2] \\
& & - Used for fast treatment \\
\hline
\end{tabular}


Table 7 Summary of avoidance and management for COVID-19

\begin{tabular}{llll}
\hline Authors & Database & Method & Accuracy \\
\hline Zhongyuan Wang et al. [105] & & Deep learning (recognition of masked face) & $95.00 \%$ \\
Joshua M. Pearce [74] & - & -open-source microcontrollers & - \\
& & -3 printers & - \\
W. Chiu et al. [21] & 72327 patient & - - infrared thermography & $90.00 \%$ \\
Edouard A. Hay [37] & - & -CNN & \\
\hline
\end{tabular}

(ii) Person Privacy Security- The tracking application of COVID-19, individual privacy security is very necessary. The government benefits from tracking the person location using the data location of mobile but this explication depends on user privacy. The security and privacy issues must be taken care of by governments [25].

(iii) Lack of dataset- A crucial challenge in a dataset like infection, medical supply, and affected areas. Currently, the dataset is from the patient collection [23, 66], social media [19], not ample for computer vision.

\subsubsection{Future work}

Several attempts have been made for COVID-19 using computer vision (machine learning, deep learning, artificial intelligence), but these are not sufficient for the diagnosis of coronavirus.

(i) Image acquisition using artificial intelligence proves to be effective and efficient in scanning. For applications in clinics, the number of the dataset must be further improved.

Table 8 Summary of lessons to be learned from COVID-19

\begin{tabular}{|c|c|c|c|}
\hline $\begin{array}{l}\text { Current } \\
\text { Response }\end{array}$ & Development & Issue & Learning Marks \\
\hline $\begin{array}{l}\text { Shortfall of } \\
\text { clarity }\end{array}$ & Initially identified by clinics & Information delay of cases & $\begin{array}{l}\text { Build betrayer for the global } \\
\text { necessity }\end{array}$ \\
\hline $\begin{array}{l}\text { Travel } \\
\text { Control }\end{array}$ & $\begin{array}{l}\text { Initially scramming for the } \\
\text { outbreak at international } \\
\text { borders }\end{array}$ & $\begin{array}{l}\text { Traveling without screening } \\
\text { through international } \\
\text { airports }\end{array}$ & $\begin{array}{l}\text { Earlier traveling from } \\
\text { high-risk countries must be } \\
\text { restricted. }\end{array}$ \\
\hline $\begin{array}{l}\text { Quarantine } \\
\text { Control }\end{array}$ & $\begin{array}{l}\text { Firstly, reported on Dec 31, } \\
\text { 2019in Wuhan }\end{array}$ & $\begin{array}{l}\text { Spread of coronavirus } \\
\text { nationally and } \\
\text { internationally }\end{array}$ & $\begin{array}{l}\text { The high-risk area must be } \\
\text { quarantine }\end{array}$ \\
\hline $\begin{array}{l}\text { Misreported } \\
\text { Public }\end{array}$ & $\begin{array}{l}\text { Falsehood opinion, falsity } \\
\text { spread among the public }\end{array}$ & $\begin{array}{l}\text { False precautions, } \\
\text { Segregation }\end{array}$ & $\begin{array}{l}\text { To escape falsity, } \\
\text { transparency must be } \\
\text { maintained. }\end{array}$ \\
\hline $\begin{array}{l}\text { Emergency } \\
\text { Notice } \\
\text { delay }\end{array}$ & $\begin{array}{l}\text { Delay of a month announcing } \\
\text { for public emergency }\end{array}$ & $\begin{array}{l}\text { Acerbity was not properly } \\
\text { broadcasted }\end{array}$ & $\begin{array}{l}\text { Development of framework } \\
\text { timely. }\end{array}$ \\
\hline $\begin{array}{l}\text { Exploration } \\
\text { and } \\
\text { Evolution }\end{array}$ & $\begin{array}{l}\text { Lack of funds for treatment and } \\
\text { vaccine of coronavirus }\end{array}$ & $\begin{array}{l}\text { Around } 3,00,000 \text { patients } \\
\text { died worldwide. }\end{array}$ & $\begin{array}{l}\text { The requirement of more } \\
\text { investment for efficient } \\
\text { treatment }\end{array}$ \\
\hline
\end{tabular}




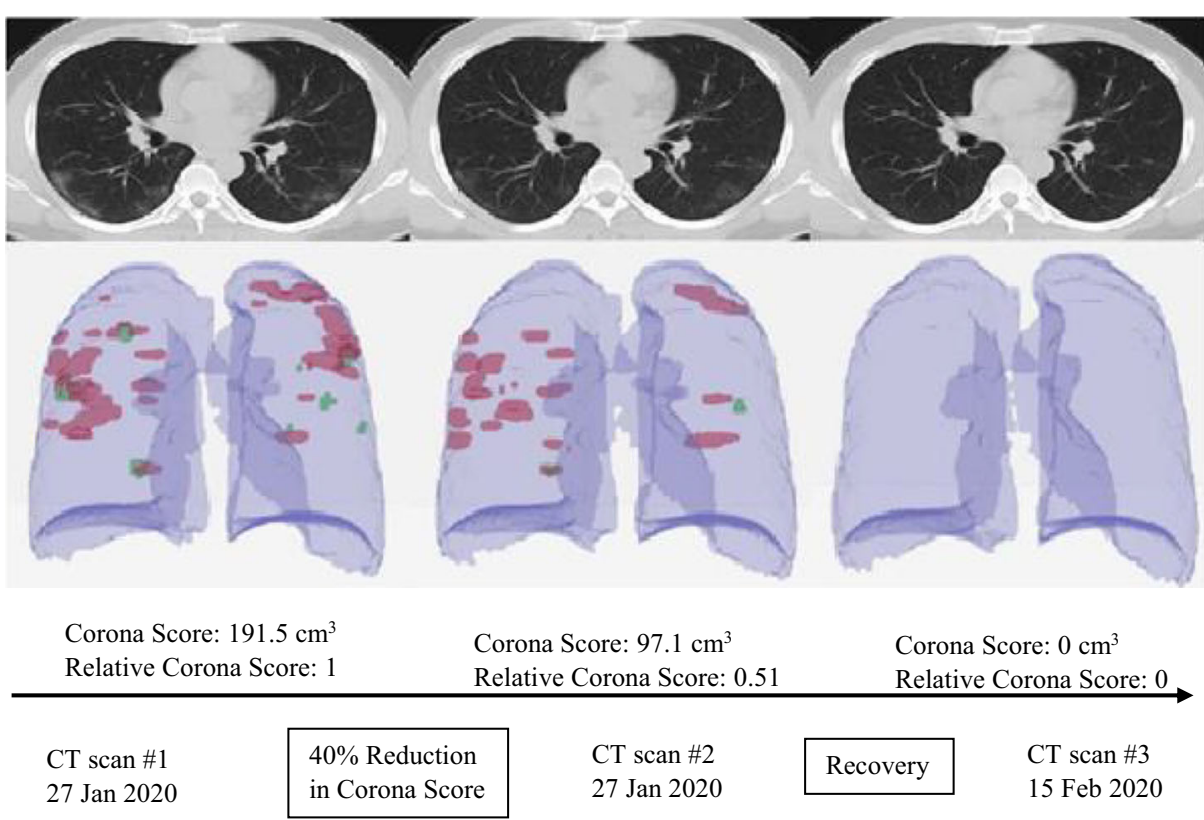

Fig. 8 Summary of Corona Score

(ii) XAI (Explainable Artificial Intelligence) [7, 29], CAM (Conventional Class Activation Mapping) are also prompted for practice in clinics.

(iii) Supervised Deep Learning [26, 72], deep learning transfer methods [95] are also incorporated for the investigation of COVID-19.

Despite limited solutions, a methodology based on machine learning [36, 57, 59, 114], medical imaging [102], fusion and oncology, Natural language processing [118], and different learning algorithms [14, 32, 48-56, 75, 76, 81, 87, 119-121, 125] could be used for measuring the coronavirus COVID-19 disease.

\section{Conclusion}

This paper presents comprehensive reviews of machine learning methods to encounter the COVID-19 (Coronavirus) epidemic. The methods are described in four categories. A

Table 9 Summary of Infection syndrome and medication

\begin{tabular}{lll}
\hline Authors & Method & Connotation \\
\hline $\begin{array}{lll}\text { Daniel Wrapp et al. [113] } \\
\text { Ophir Gozes et al. [34] }\end{array}$ & - Biophysical assays & - Trimeric Spike glycoprotein is used to bind virus \\
& - Based on CT scan images \\
Yumlu Wang et al. [107] & - Deep learning & -191.5 com3 is measured \\
& - Bidirectional Neural & - Classification of abnormal respiratory \\
Yoshihiro Uesawa et al. & - Detwork learning & \\
[99] & & - Drug discovery \\
\hline
\end{tabular}


comprehensive summary of classical approaches that includes resources for development and research is presented. The article survey on various image processing methods with wide bibliography for coronavirus results in new research encouragement. Yet, this survey was favored as an early review for testing and controlling the COVID-19 pandemic. These attempts impact the outbreak and post coronavirus environment.

Funding This research is not having any specific grant from funding agencies in the public, commercial, or not for profit sectors.

Declaration Not Applicable.

Conflict of interest Authors do not have any conflicts.

\section{References}

1. Abbas A, Abdelsamea MM, Gaber MM (2020) Classification of COVID-19 in chest X-ray images using DeTraC deep convolutional neural network. arXiv preprint arXiv:2003.13815

2. AI Helps Doctors Diagnose the Coronavirus. (n.d.) [Online]. Available: https://news.developer.nvidia. com/ai-helps-doctors-diagnose-thecoronavirus/

3. Ai T, Yang Z, Hou H, Zhan C, Chen C, Lv W, Tao Q, Sun Z, Xia L (2020) Correlation of chest CT and RT-PCR testing in coronavirus disease 2019 (COVID-19) in China: a report of 1014 cases. Radiology 26: 200642

4. Al-antari MA, Al-masni MA, Choi MT, Han SM, Kim TS (2018) A fully integrated computer-aided diagnosis system for digital X-ray mammograms via deep learning detection, segmentation, and classification. Int J Med Inform 117:44-54

5. Amin SU, Hossain MS, Muhammad G, Alhussein M, Rahman MA (2019) Cognitive smart healthcare for pathology detection and monitoring. IEEE Access 7:10745-10753

6. Apostolopoulos ID, Mpesiana TA (2020) Covid-19: automatic detection from X-ray images utilizing transfer learning with convolutional neural networks. Australas Phys Eng Sci Med 43:635-640

7. Arrieta AB, Díaz-Rodríguez N, Del Ser J, Bennetot A, Tabik S, Barbado A et al (2020) Explainable artificial intelligence (XAI): concepts, taxonomies, opportunities and challenges toward responsible AI. Information Fusion 58:82-115

8. Asnaoui KE, Chawki Y, Idri A (2020) Automated Methods for Detection and Classification Pneumonia based on X-Ray Images Using Deep Learning. arXiv preprint arXiv:2003.14363

9. Barstugan M, Ozkaya U, Ozturk S (2020) Coronavirus (COVID-19) Classification using CT Images by Machine Learning Methods. arXiv preprint arXiv:2003.09424

10. Bishop CM (2006) Pattern recognition and machine learning. Springer

11. BlueDot: Outbreak Risk Software. (n.d.) [Online]. Available: https://bluedot.global/

12. Can CCTV (n.d.) help contain the Coronavirus?. [Online]. Available: https://www.ifsecglobal.com/asia/ can-cctv-help-contain-coronavirus/.

13. Cao Y, Xu Z, Feng J, Jin C, Han X, Wu H et al (2020) Longitudinal assessment of COVID-19 using a deep learning-based quantitative CT pipeline: illustration of two cases. Radiol Cardiothorac Imaging 2:e200082

14. Chen Y, Xiong J, Xu W, Zuo J (2019) A novel online incremental and decremental learning algorithm based on variable support vector machine. Clust Comput 22(3):7435-7445

15. Chen Y, Liu Q, Guo D (2020) Emerging coronaviruses: genome structure, replication, and pathogenesis. J Med Virol 92(4):418-423

16. Chen R, Chen J, Meng QT (2020) Chest computed tomography images of early coronavirus disease (COVID-19). Can J Anesth/J Can d'anesthésie 11:1-2

17. Chen J, Wu L, Zhang J, Zhang L, Gong D, Zhao Y, Hu S, Wang Y, Hu X, Zheng B, Zhang K (2020) Deep learning-based model for detecting 2019 novel coronavirus pneumonia on high-resolution computed tomography: a prospective study. medRxiv

18. Chen J, Wu L, Zhang J, Zhang L, Gong D, Zhao Y, et al. (2020) Deep learning-based model for detecting 2019 novel coronavirus pneumonia on high-resolution computed tomography: a prospective study. MedRxiv 
19. Chen E, Lerman K, Ferrara E (2020) Covid-19: The first public coronavirus twitter dataset," arXiv preprint arXiv:2003.07372

20. Chen C, Gao G, Xu Y, Pu L, Wang Q, Wang L, Wang W, Song Y, Chen M, Wang L, Yu F (n.d.) SARSCoV-2-positive sputum and feces after conversion of pharyngeal samples in patients with COVID-19. Ann Intern Med

21. Chiu WT, Lin PW, Chiou HY, Lee WS, Lee CN, Yang YY, Lee HM, Hsieh MS, Hu CJ, Ho YS, Deng WP (2005) Infrared thermography to mass-screen suspected SARS patients with fever. Asia Pac J Public Health 17(1):26-28

22. Chowdhury MEH, Rahman T, Khandakar A, Mazhar R, Kadir MA, Mahbub ZB, Islam KR, Khan MS, Iqbal A, Al-Emadi N, Reaz MBI (2020) Can AI help in screening viral and COVID-19 pneumonia? arXiv preprint

23. Cohen JP, Morrison P, Dao L (2020) COVID-19 image data collection. arXiv preprint arXiv:2003.11597

24. Computational predictions of protein structures associated with COVID-19. (n.d.) [Online]. Available: https://deepmind.com/research/opensource/computational-predictions-of-protein-structures-associated with-COVID-19

25. Coronavirus-tracking smartphone apps don't invade privacy says data watchdog. (n.d.) [Online]. Available: https:/www.zdnet.com/article/coronavirus-tracking-mobile-appsget-green-light-from-data-watchdog/

26. Doersch C, Gupta A, Efros AA (2015) Unsupervised visual representation learning by context prediction. In: Proceedings of the IEEE International Conference on Computer Vision, pp. 1422-1430.

27. Erickson BJ, Korfiatis P, Akkus Z, Kline TL (2017) Machine learning for medical imaging. Radiographics 37(2):505-515

28. Farooq M, Hafeez A (2020) COVID-ResNet: A Deep Learning Framework for Screening of COVID19 from Radiographs. arXiv preprint arXiv:2003.14395

29. Fellous J-M, Sapiro G, Rossi A, Mayberg HS, Ferrante M (2019) Explainable artificial intelligence for neuroscience: behavioural neurostimulation. Front Neurosci 13:1346

30. Five Companies Using AI to Fight Coronavirus. (n.d.) [Online]. Available: https://spectrum.ieee.org/thehuman-os/artificial-intelligence/medicalai/companies-ai-coronavirus

31. Gaál G, Maga B, Lukács A (2020) Attention U-Net Based Adversarial Architectures for Chest X-ray Lung Segmentation. arXiv preprint arXiv:2003.10304

32. Gadekallu TR, Rajput DS, Reddy MPK, Lakshmanna K, Bhattacharya S, Singh S, Jolfaei A, Alazab M (2020) A novel PCA-whale optimization-based deep neural network model for classification of tomato plant diseases using GPU. J Real-Time Image Process 1-14

33. Ghoshal B, Tucker A (2020) Estimating Uncertainty and Interpretability in Deep Learning for Coronavirus (COVID-19) Detection. arXiv preprint arXiv:2003.10769

34. Gozes O, Frid-Adar M, Greenspan H, Browning PD, Zhang H, Ji W, Bernheim A, Siegel E (2020) Rapid ai development cycle for the coronavirus (covid-19) pandemic: Initial results for automated detection \& patient monitoring using deep learning ct image analysis. arXiv preprint arXiv:2003.05037

35. Gozes O, Frid-Adar M, Greenspan H, Browning PD, Zhang H, Ji W, et al. (2020) Rapid AI development cycle for the coronavirus (covid-19) pandemic: Initial results for automated detection \& patient monitoring using deep learning ct image analysis. arXiv:2003.05037

36. Hao J, Kim Y, Mallavarapu T, Oh JH, Kang M (2019) Interpretable deep neural network for cancer survival analysis by integrating genomic and clinical data. BMC Med Genet 12:1-13

37. Hay EA, Parthasarathy R (2018) Performance of convolutional neural networks for identification of bacteria in 3D microscopy datasets. PLoS Comput Biol 14(12):e1006628

38. Hemdan EE, Shouman MA, Karar ME (2020) COVIDX-Net: A Framework of Deep Learning Classifiers to Diagnose COVID-19 in X-Ray Images. arXiv preprint arXiv:2003.11055

39. Hölbl M, Kompara M, Kamišali'c A, Zlatolas LN (2018) A systematic review of the use of blockchain in healthcare. Symmetry 10(10):470

40. Huang L, Han R, Ai T, Yu P, Kang H, Tao Q et al (2020) Serial quantitative chest CT assessment of COVID-19: deep-learning approach. Radiol Cardiothorac Imaging 2:e200075

41. Infervision in the Frontlines Against the Coronavirus. (n.d.) [Online]. Available: https:/www.infervision.com/

42. Jiang F, Jiang Y, Zhi H, Dong Y, Li H, Ma S, Wang Y, Dong Q, Shen H, Wang Y (2017) Artificial intelligence in healthcare: past, present and future. Stroke Vasc Neurol 2(4):230-243

43. Jin C, Chen W, Cao Y, Xu Z, Zhang X, Deng L, Zheng C, Zhou J, Shi H, Feng J (2020) Development and Evaluation of an AI System for COVID-19 Diagnosis. medRxiv

44. Jin S, Wang B, Xu H, Luo C, Wei L, Zhao W, Hou X, Ma W, Xu Z, Zheng Z, Sun W (2020) AI-assisted CT imaging analysis for COVID-19 screening: Building and deploying a medical AI system in four weeks. medRxiv

45. Jin S, Wang B, Xu H, Luo C, Wei L, Zhao W, et al. (2020) AI-assisted CT imaging analysis for COVID19 screening: building and deploying a medical AI system in four weeks. MedRxiv 
46. Jin C, Cheny W, Cao Y, Xu Z, Zhang X, Deng v, et al. (2020) Development and evaluation of an AI system for COVID-19 diagnosis. MedRxiv

47. Kanwal N, Girdhar A, Gupta S (2011) Region based adaptive contrast enhancement of medical X-ray images. In: 2011 5th international conference on bioinformatics and biomedical engineering 2011 May 10 (pp. 1-5). IEEE

48. Khan MA, Sharif M, Akram T, Bukhari SAC, Nayak RS (2019) Developed Newton-Raphson based deep features selection framework for skin lesion recognition. Pattern Recognit Lett. https://doi.org/10.1016/j. patrec.2019.11.034.

49. Khan MA, Sharif M, Akram T, Yasmin M, Nayak RS (2019) Stomach deformities recognition using rankbased deep features selection. J Med Syst 43:329. https://doi.org/10.1007/s10916-019-1466-3

50. Khan MA, Rashid M, Sharif M, Javed K, Akram T (2019) Classification of gastrointestinal diseases of stomach from WCE using improved saliency-based method and discriminant features selection. Multimed Tools Appl 78:27743-27770. https://doi.org/10.1007/s11042-019-07875-9

51. Khan MA, Lali IU, Rehman A, Ishaq M, Sharif M, Saba T, Zahoor S, Akram T (2019) Brain tumor detection and classification: a framework of marker-based watershed algorithm and multilevel priority features selection. Microsc Res Tech 909-922

52. Khan MA, Akram T, Sharif M, Saba T, Javed K, Lali IU, Tanik UJ, Rehman A (2019) Construction of saliency map and hybrid set of features for efficient segmentation and classification of skin lesion. Microsc Res Tech 741-763

53. Khan SA, Nazir M, Khan MA, Saba T, K Javed, Rehman A, Akram T, Awais M (2019) Lungs nodule detection framework from computed tomography images using support vector machine. Microsc Tech 1256-1266

54. Khan MA, Akram T, Sharif M, Javed K, Rashid M, Bukhari SAC (2020) An integrated framework of skin lesion detection and recognition through saliency method and optimal deep neural network features selection. Neural Comput Applic 32:15929-15948. https://doi.org/10.1007/s00521-019-04514-0

55. Khana MA, Rubab S, Kashif A, Sharif MI, Muhammad N, Shahd JH, Zhange Y-D, Satapathy SC (2020) Lungs cancer classification from CT images: an integrated design of contrast based classical features fusion and selection. Pattern Recogn Lett 129:77-85

56. Khare N, Devan P, Chowdhary CL, Bhattacharya S, Singh G, Singh S, Yoon B (2020) SMO-DNN: Spider Monkey Optimization and Deep Neural Network Hybrid Classifier Model for Intrusion Detection. Electronics 9(4):692

57. Kim DW, Lee SH, Kwon S, Nam W, Cha I, Kim HJ (2019) Deep learning-based survival prediction of oral cancer patients. Sci Rep 9:6994

58. Kopaczka M, Kolk R, Merhof D (2018) A fully annotated thermal face database and its application for thermal facial expression recognition. In: 2018 IEEE international instrumentation and measurement technology conference (I2MTC) 2018 May 14 (pp. 1-6). IEEE

59. Kourou K, Exarchos TP, Exarchos KP, Karamouzis MV, Fotiadis DI (2015) Machine learning applications in cancer prognosis and prediction. Comput Struct Biotechnol J 13:8-17

60. Lai C-C, Shih T-P, Ko W-C, Tang H-J, Hsueh P-R (2020) Severe acute respiratory syndrome coronavirus 2 (sars-cov-2) and corona virus disease-2019 (covid-19): the epidemic and the challenges. Int J Antimicrob Agents 55(3):105924

61. Li Y, Xia L (2020) Coronavirus disease 2019 (COVID-19): role of chest CT in diagnosis and management. Am J Roentgenol 21:1-7

62. Li L, Qin L, Xu Z, Yin Y, Wang X, Kong B, Bai J, Lu Y, Fang Z, Song Q, Cao K (2020) Artificial intelligence distinguishes COVID-19 from community acquired pneumonia on chest CT. Radiology 200905

63. Li L, Qin L, Xu Z, Yin Y, Wang X, Kong B, et al. (2020) Artificial intelligence distinguishes COVID-19 from community acquired pneumonia on chest CT. Radiology 200905

64. G. Litjens, T. Kooi, B. E. Bejnordi, A. A. A. Setio, F. Ciompi, M. Ghafoorian, J. A. Van Der Laak, B. Van Ginneken, and C. I. S'anchez, "A survey on deep learning in medical image analysis," Med Image Anal, vol. 42, pp. 60-88, 2017.

65. Liu T, Huang P, Liu H, Huang L, Lei M, Xu W, Hu X, Chen J, Liu B (2020) Spectrum of chest CT findings in a familial cluster of COVID-19 infection. Radiol Cardiothorac Imaging 2(1):e200025

66. Liu W, Yen PT-W, Cheong SA (2020) Coronavirus disease 2019 (covid-19) outbreak in china, spatial temporal dataset," arXiv preprint arXiv:2003.11716

67. Lu H, Stratton CW, Tang Y (2020) Outbreak of pneumonia of unknown etiology in Wuhan China: the mystery and the miracle. J Med Virol 25678

68. Miotto R, Wang F, Wang S, Jiang X, Dudley JT (2018) Deep learning for healthcare: review, opportunities and challenges. Brief Bioinform 19(6):1236-1246 
69. Monrat AA, Schelén O, Andersson K (2019) A survey of blockchain from the perspectives of applications, challenges, and opportunities. IEEE Access 7:117134-117151

70. Narin A, Kaya C, Pamuk Z 2020 Automatic Detection of Coronavirus Disease (COVID-19) Using X-ray Images and Deep Convolutional Neural Networks. arXiv preprint arXiv:2003.10849

71. Nguyen DC, Ding M (2020) Blockchain and AI based solutions to combat coronavirus (COVID-19)- like Epidmics: A Survey. https://doi.org/10.36227/techrxiv.12121962

72. Noroozi M, Vinjimoor A, Favaro P, Pirsiavash H (2018) Boosting self-supervised learning via knowledge transfer. In: Proceedings of the IEEE Conference on Computer Vision and Pattern Recognition, pp. 93599367.

73. Paules CI, Marston HD, Fauci AS (2020) Coronavirus infections - more than just the common cold. JAMA 323(8):707-708

74. Pearce JM (2020) A review of open source ventilators for COVID-19 and future pandemics. F1000Research 9(218):218

75. Peng F, Lei Y-z, Long M, Sun X-m (2011) A reversible watermarking scheme for two-dimensional CAD engineering graphics based on improved difference expansion. Comput Aided Des 43(8):1018-1024

76. Peng F, Liu Y, Long M (2014) Reversible watermarking for 2D CAD engineering graphics based on improved histogram shifting. Comput Aided Des 49:42-50

77. Pisani P, Renna MD, Conversano F, Casciaro E, Muratore M, Quarta E, Di Paola M, Casciaro S (2013) Screening and early diagnosis of osteoporosis through X-ray and ultrasound based techniques. World J Radiol 5(11):398-410

78. Pouyanfar S, Sadiq S, Yan Y, Tian H, Tao Y, Reyes MP, Shyu M-L, Chen S-C, Iyengar S (2018) A survey on deep learning: algorithms, techniques, and applications. ACM Comput Surv (CSUR) 51(5):1-36

79. Qi X, Jiang Z, Yu Q, Shao C, Zhang H, Yue H, et al. (2020) Machine learning-based CT radiomics model for predicting hospital stay in patients with pneumonia associated with SARS-CoV-2 infection: a multicenter study. MedRxiv

80. Reddy S, Fox J, Purohit MP (2019) Artificial intelligence-enabled healthcare delivery. J R Soc Med 112(1):22-28

81. Saba T, Khan MA, Rehman A, Marie-Sainte SL (2019) Region extraction and classification of skin cancer: a heterogeneous framework of deep CNN features fusion and reduction. J Med Syst 43:289. https://doi. org/10.1007/s10916-019-1413-3

82. Sarker IH (2019) Context-aware rule learning from smartphone data: survey, challenges and future directions. J Big Data 6(1):95

83. Sarker IH, Kayes A, Watters P (2019) Effectiveness analysis of machine learning classification models for predicting personalized context-aware smartphone usage. J Big Data 6(1):57

84. Sethy PK, Behera SK (n.d.) Detection of Coronavirus disease (COVID-19) based on deep features

85. Shan F, Gao Y, Wang J, Shi W, Shi N, Han M, Xue Z, Shen D, Shi Y (2020) Lung Infection Quantification of COVID-19 in CT Images with Deep Learning. arXiv preprint arXiv:2003.04655

86. Shan F, Gao Y, Wang J, Shi W, Shi N, Han M, et al. (2020) Lung infection quantification of COVID-19 in CT images with deep learning. arXiv:2003.04655

87. Sharif MI, Li JP, Khan MA, Saleem MA (2019) Active Deep neural Network Features Selection for Segmentation and Recognition of Brain Tumors using MRI Images. Pattern Recognit Lett. https://doi.org/ 10.1016/j.patrec.2019.11.019

88. Shen J, Shafiq MO (2019) Learning mobile application usage-a deep learning approach. In: 2019 18th IEEE International Conference On Machine Learning and Applications (ICMLA),( pp. 287-292)

89. Shen C, Yu N, Cai S, Zhou J, Sheng J, Liu K, et al. (2020) Quantitative computed tomography analysis for stratifying the severity of coronavirus disease 2019. J Pharm Anal

90. Shi H, Han X, Jiang N, Cao Y, Alwalid O, Gu J, Fan Y, Zheng C (2020) Radiological findings from 81 patients with COVID-19 pneumonia in Wuhan, China: a descriptive study. Lancet Infect Dis

91. Shi F, Xia L, Shan F, Wu D, Wei Y, Yuan H, et al. (2020) Large-scale screening of COVID-19 from community acquired pneumonia using infection size-aware classification. arXiv:2003.09860

92. Shrestha A, Mahmood A (2019) Review of deep learning algorithms and architectures. IEEE Access 7: 53040-53065

93. Song Y, Zheng S, Li L, Zhang X, Zhang X, Huang Z, Chen J, Zhao H, Jie Y, Wang R, Chong Y (2020) Deep learning Enables Accurate Diagnosis of Novel Coronavirus (COVID-19) with CT images. medRxiv

94. Speidel MA, Wilfley BP, Star-Lack JM, Heanue JA, Van Lysel MS (2006) Scanning-beam digital x-ray (SBDX) technology for interventional and diagnostic cardiac angiography. Med Phys 33(8):2714-2727

95. Tan C, Sun F, Kong T, Zhang W, Yang C, Liu C (2018) A survey on deep transfer learning. In: International Conference on Artificial Neural Networks (pp. 270-279)

96. Tang L, Zhang X, Wang Y, Zeng X (2020) Severe COVID-19 pneumonia: assessing inflammation burden with volume-rendered chest CT. Radiol Cardiothorac Imaging 2:e200044 
97. Tang Z, Zhao W, Xie X, Zhong Z, Shi F, Liu J, et al. (2020) Severity assessment of coronavirus disease 2019 (COVID-19) using quantitative features from chest CT images. arXiv:2003.11988

98. The role of wireless tech in fighting the new coronavirus. (n.d.) [Online]. Available: https://www. lightreading.com/4g-3g-wifi/the-role-ofwireless-tech-in-fighting-the-new-coronavirus/d/d-id/757634

99. Uesawa Y (2018) Quantitative structure-activity relationship analysis using deep learning based on a novel molecular image input technique. Bioorg Med Chem Lett 28(20):3400-3403

100. Wang L, Wong A (2020) COVID-Net: A Tailored Deep Convolutional Neural Network Design for Detection of COVID-19 Cases from Chest Radiography Images. arXiv preprint arXiv:2003.09871

101. Wang X, Peng Y, Lu L, Lu Z, Bagheri M, Summers RM (2017) Chestx-ray8: Hospital-scale chest X-ray database and benchmarks on weakly-supervised classification and localization of common thorax diseases. In: Proceedings of the IEEE conference on computer vision and pattern recognition (pp. 2097-2106)

102. Wang X, Peng Y, Lu L, Lu Z, Summers RM (2018) TieNet: Text-image embedding network for common thorax disease classification and reporting in chest X-rays. In: Proceedings of the IEEE Conference on Computer Vision and Pattern Recognition (pp. 9049-9058)

103. Wang W, Xu Y, Gao R, Lu R, Han K, Wu G, Tan W (2020) Detection of SARS-CoV-2 in different types of clinical specimens. JAMA

104. Wang S, Kang B, Ma J, Zeng X, Xiao M, Guo J, Cai M, Yang J, Li Y, Meng X, Xu B (2020) A deep learning algorithm using CT images to screen for Corona Virus Disease (COVID-19). medRxiv

105. Wang Z, Wang G, Huang B, Xiong Z, Hong Q, Wu H, Yi P, Jiang K, Wang N, Pei Y, Chen H (2020) Masked Face Recognition Dataset and Application. arXiv preprint arXiv:2003.09093

106. Wang S, Kang B, Ma J, Zeng X, Xiao M, Guo J, et al.(2020) A deep learning algorithm using CT images to screen for Corona virus disease (COVID-19). MedRxiv

107. Wang Y, Hu M, Li Q, Zhang XP, Zhai G, Yao N (2020) Abnormal respiratory patterns classifier may contribute to large-scale screening of people infected with COVID-19 in an accurate and unobtrusive manner. arXiv preprint arXiv:2002.05534

108. AI Weekly (n.d.) Coronavirus, facial recognition, and the future of privacy. [Online]. Available: https:// venturebeat.com/2020/03/06/aiweekly-coronavirus-facial-recognition-and-the-future-of-privacy/.

109. WHO (April 10, 2020) Coronavirus disease 2019 (COVID-19) Situation Report - 80. Available: https:// www.who.int/docs/default-source/coronaviruse/situation-reports/20200409-sitrep-80-covid-19.pdf? sfvrsn=1b685d64 4. Accessed 3 May 2020

110. WHO (30 January, 2020) Statement on the second meeting of the International Health Regulations (2005) Emergency Committee regarding the outbreak of novel coronavirus (2019-nCoV). Accessed 3 May 2020

111. WHO (2020) WHO Director-General's opening remarks at the media briefing on COVID-19

112. World Health Organization (2020) Rational use of personal protective equipment (PPE) for coronavirus disease (COVID-19): interim guidance, 19 March 2020. World Health Organization

113. Wrapp D, Wang N, Corbett KS, Goldsmith JA, Hsieh CL, Abiona O, Graham BS, McLellan JS (2020) Cryo-EM structure of the 2019-nCoV spike in the prefusion conformation. Science. 367(6483):1260-1263

114. Xu Y, Hosny A, Zeleznik R, Parmar C, Coroller TP, Franco I et al (2019) Deep learning predicts lung cancer treatment response from serial medical imaging. Clin Cancer Res 25:3266-3275

115. Xu X, Jiang X, Ma C, Du P, Li X, Lv S, Yu L, Chen Y, Su J, Lang G, Li Y (2020) Deep learning system to screen coronavirus disease 2019 pneumonia. arXiv preprint arXiv:2002.09334

116. Xu X, Jiang X, Ma C, Du P, Li X, Lv S, et al. (2020) Deep learning system to screen Coronavirus disease 2019 pneumonia. arXiv:2002.09334

117. Ying S, Zheng S, Li L, Zhang X, Zhang X, Huang Z et al. (2020) Deep learning enables accurate diagnosis of novel coronavirus (COVID-19) with CT images. MedRxiv

118. Yuan J, Liao H, Luo R, Luo J (2019) Automatic radiology report generation based on multi-view image fusion and medical concept enrichment. In: International Conference on Medical Image Computing and Computer-Assisted Intervention, pp. 721-729

119. Zhang D, Li Q, Yang G, Li L, Sun X (2017) Detection of image seam carving by using weber local descriptor and local binary patterns. J Inf Secur Appl 36:135-144

120. Zhang D, Yin T, Yang G, Xia M, Li L, Sun X (2017) Detecting image seam carving with low scaling ratio using multi-scale spatial and spectral entropies. J Vis Commun Image Represent 48:281-291

121. Zhang L-B, Peng F, Qin L, Long M (2018) Face spoofing detection based on color texture Markov feature and support vector machine recursive feature elimination. J Vis Commun Image Represent 51:56-69

122. Zhang J, Xie Y, Li Y, Shen C, Xia Y (2020) COVID-19 screening on Chest X-ray images using deep learning based anomaly detection. arXiv:2003.12338

123. Zhao J, Zhang Y, He X, Xie P (2020) COVID-CT-Dataset: A CT Scan Dataset about COVID-19. arXiv preprint arXiv:2003.13865

124. Zheng C, Deng X, Fu Q, Zhou Q, Feng J, Ma H, et al. (2020) Deep learning-based detection for COVID19 from chest CT using weak label. MedRxiv 
125. Zhou S, Tan B (2020) Electrocardiogram soft computing using hybrid deep learning CNN-ELM. Appl Soft Comput 86:105778

Publisher's note Springer Nature remains neutral with regard to jurisdictional claims in published maps and institutional affiliations. 